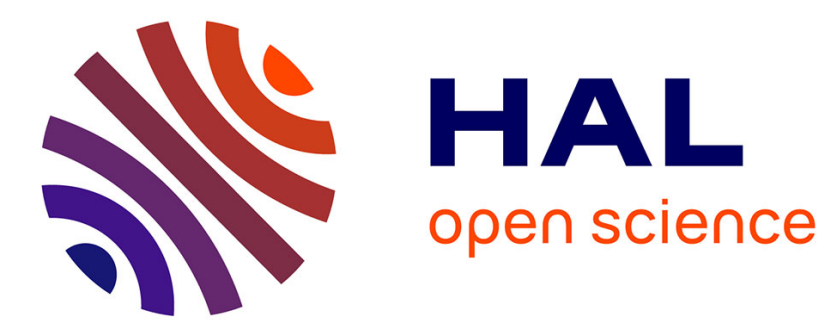

\title{
Le refus du dialogue : les sonnets 37-49 de Guittone d'Arezzo
}

\author{
Anne Robin
}

\section{To cite this version:}

Anne Robin. Le refus du dialogue : les sonnets 37-49 de Guittone d'Arezzo. Arzanà. Cahiers de littérature médiévale italienne, 2003, Les voix multiples. Du conflit au dialogue. Etudes réunies par M. Marietti \& C. Perrus., 9, p. 43-64. hal-01395720

\section{HAL Id: hal-01395720 https://hal.univ-lille.fr/hal-01395720}

Submitted on 11 Nov 2016

HAL is a multi-disciplinary open access archive for the deposit and dissemination of scientific research documents, whether they are published or not. The documents may come from teaching and research institutions in France or abroad, or from public or private research centers.
L'archive ouverte pluridisciplinaire HAL, est destinée au dépôt et à la diffusion de documents scientifiques de niveau recherche, publiés ou non, émanant des établissements d'enseignement et de recherche français ou étrangers, des laboratoires publics ou privés. 


\section{ARZANÀ 9}

\section{Les voix multiples}

du conflit au dialogue

ÉTUdES RÉUNies ET PRÉSENTEEES par M.MarietTt et C.Perrus

UNIVERSITE dE LA SORbONNE NOUVElLE U.E.R D'ITALIEN ET DE ROUMAIN

Centre d'Études ET de Recherches SUR

La Litterature ItalienNe MEdievale (CERLIM) 


\section{TABLE DES MATIERES}

Avant-propos

Véronique ABBRUZZETTI,

Une controverse médiévale :

la disputatio Musce cum formica de Bonvesin da Ripa ....13

Anne ROBIN,

Le refus du dialogue :

les sonnets 37-49 de Guittone d'Arezzo

Claude PERRUS,

Rites du dialogue courtois chez Monte Andrea 65

Johannes BARTUSCHAT,

La tenson avec le Dieu d'Amour de Monte Andrea ...... 77

Cécile LE LAY

Le contrasto en forme de procès

chez Jacopone da Todi

Marina MARIETTI

La «bataille " des pensées

et l'accord des voix dans la Vita Nova

Claire CABAILLOT,

Le Secretum : dialogue éristique ou heuristique ? ..........151

\section{Donatella BISCONTI}

Le dialogue " absent " dans la première

églogue de Francesco Arzocchi

\section{Nella BIANCHI BENSIMON,}

Une ou plusieurs voix?

La construction dialogique dans la Vita Civile

de Matteo Palmieri

Michelle SCHULLER,

La correspondance de la famille Strozzi :

l'écriture épistolaire comme discours de l'absence ......243 


\section{LE REFUS DU DIALOGUE}

Les sonnets 37-49 de Guittone d'Arezzo

I a tenson qui occupe les sonnets 37-49 de Guittone Ld'Arezzo' est singulière à plus d'un titre. Avec celle plus connue qui oppose la villana donna et le villan parladore (81-86) elle fonde un modèle qui adapte à la forme italienne du sonnet la tradition provençale de la tenson d'amour entre l'amant et sa dame: les deux amants, ici fictifs, utilisent tour à tour l'espace d'un sonnet pour exposer leurs arguments et détruire ou accepter ceux de leur interlocuteur.

Tout en constituant d'autre part, comme toutes les tensons, un ensemble fini qui peut être considéré isolément $^{2}$, la macro-unité 37-49 appartient à une structure plus vaste comptant 86 sonnets que Lino Leonardi a intitulée canzoniere après avoir mis en évidence son unité formelle et narrative. Dans ce contexte où un amant nommé Guittone tente de séduire de manière courtoise et loyale la dame qu'il aime, notre tenson vient après six sonnets de louange adressés à Gioia - senhal de la dame à la fin desquels l'amant se résout à formuler une déclaration et une requête d'amour qui occupent le premier sonnet du dialogue.

Cette tenson est singulière enfin en raison de sa structure. Malgré une unité d'ensemble assurée par la thématique - une déclaration d'amour visant à obtenir de la dame l'octroi d'un rendez-vous dans un lieu écarté - et par de très nombreuses connexions d'ecriture, elle se divise en deux parties très différentes. Tout d'abord le cialogue ne 
procède pas de manière progressive entre le sonnet 37 et le sonnet 49 , mais progresse jusqu'au sonnet central 43 a partir duquel il régresse: jusqu'à 43 en effet la dame se montre bienveillante et laisse à Guittone une chance de la séduire, puis elle devient hostile et repousse tous les arguments de son amant. Ce changement est d'ailleurs flagrant, tant parce que la dame ne cesse d'enjoindre à Guittone d'abandonner la partie au moyen d'un verbe qui revient comme un leitmotiv dans tous les sonnets où elle prend la parole après 43 - "Consiglioti che parti " et "Parteti " (44, en tête des v.1 et v. 9); " parteti ormai! " (46, v. 10 à la rime); "Donque te parte " (48, en tête du v. 9) que du fait de l'évolution de la tonalité des incipit de Guittone: alors que dans le sonnet 37 l'amant s'adresse encore à "Gioia gioiosa ", alors qu'il ouvre le sonnet 39 avec "Grazi'e mercé voi, gentil donna orrata ", puis exprime des sentiments mélangés dans le sonnet 41 " lo dolor e la gioi...", il commence tous les sonnets suivants par des exclamations de souffrance: "Oimè, che dite amor? " (43), "Lasso!, non sete là dov'eo tormento/ piangendo e sospirando, amor, per voi " (45) et « $\mathrm{Ai}$ ! come m'è crudel, forte e noiosa/ciascuna parte » (47)

D'autre part la bipartition s'organise autour de deux demandes de conseil : lorsque la dame consent à répondre à Guittone elle termine son allocution en lui demandant conseil à propos de l'attitude qu'elle doit adopter face à sa requête a moureuse $(38$, v. $10-11)$ et les sonnets suivants concernent cette demande; à partir de 43 les rôles sont inversés, c'est au tour de Guittone de demander à sa dame ce qu'elle lui conseille de faire face à son ordre $(43, v, 7-8)$ Sachant que la demande de conseil est un sous-genre de la tenson, in peut donc presque considérer le dialogue 37-49 comme une succession de deux tensons distinctes.

C'est la question que pose cette singularité structurelle - à savoir: qu'est-ce qui provoque le revirement de la dame? Ou: quel est le cheminement logique de ce dialogue en deux parties? - qui est à l'origine de notre étude.

$\star \star *$

Lorsque la dame répond pour la première fois à Guittone, elle reprend les deux points de sa déclaration d'amour en les accompagnant de mises en garde. La première de ces mises en garde est particulièrement importante parce qu'elle concerne la vérité du discours, vérité dont dépendra la réponse de la dame:

Tu dis que tu m'aimes beaucoup, de bon cour: mais mesure bien si ce mot est sincère,

car pour amour, amour je te rendrai,

mais, pour son contraire, ce qui est juste et équitable. ( 38 , v. $5-8)^{\text {* }}$

Cette condition non seulement est énoncée d'entrée de jeu, mais elle est sous-jacente également dans les trois derniers vers du sonnet où le qualificatif « loyal », équivalent du précédent « sincère », est d'une part répété, d'autre part mis en évidence par une construction en chiasme:

Conseille-moi, en homme loyal et sage,

sur ce que je dois faire face à ta demande,

car d'un loyal conseil je ne m'éloignerai pas. $(38, \mathrm{v} .12-14)^{-}$

Cet impératif de franchise, de sincérité et de loyauté relève en partie du code de comportement courtois et il est imposé par la dame en réponse à la courtoisie dont ellemême se prévaut dès qu'elle prend la parole (v. 1-4) ${ }^{\circ}$, mais l'insistance avec laquelle il est rappelé dépasse la topique. Il paraît constituer au fond le sujet de cette première intervention féminine et fixer les conditions du dialogue qui s'ouvre. 
En s'en tenant à ce sonnet initial on pourrait émettre l'hypothèse suivante pour expliquer le revirement de la dame après $43: c^{\prime}$ est parce qu'elle a découvert la mauvaise foi de Guittone - ce que confirme d'ailleurs le reproche qu'elle lui adresse d'être un "faux amant ", un "simulateur " (infingitore), quelqu'un qui fait en sorte de "paraître » avoir le coeur épris (44, v. 4 et 6) - qu'elle met un terme à son attitude bienveillante. Mais il faudrait ne pas tenir compte du deuxième sonnet dans lequel la dame prend la parole (40), un sonnet surprenant à bien des égards. Bien que la dame y réponde au sonnet précédent de Guittone au moyen entre autres de connexions d'écriture - elle repart sur le « conseil » (v.2) qu'il lui a donné de se fier à sa loyauté -, elle abandonne d'abord le discours allocutif pour prononcer une diatribe impersonnelle contre les conseillers fallacieux et les serviteurs infideles (v. 1-8) ' . Elle s'adresse à nouveau directement à son interlocuteur dans les tercets, en reliant les deux parties de son discours au moyen du procédé des coblas capfinidas $^{\circledR}$ suggérant ainsi que Guittone compte parmi ces conseillers fallacieux, mais en tenant des propos qui contredisent complètement ce qu'elle avait laissé entendre lors de sa première intervention:

Mais tu ne peux face à moi changer d'aspect

sans que je ne te reconnaisse clairement,

bien que je ne veuille pas pour autant renoncer

à te considérer comme un amant sincère,

selon la forme de tes paroles,

en les prenant à la lettre. (v. 9-14)

Elle dit littéralement, en somme, qu'elle sait fort bien que Guittone est hypocrite, mais qu'en dépit de cela elle va continuer à le considérer comme un amant épris, se fiant dès lors à la forme, et non au fond, de son discours (elle insiste particulièrement là-dessus au moyen de la redondance des deux derniers vers). Le préalable de la sincérité est donc ici clairement abandonné.

Avant de réfléchir à la signification qu'il faut donner à ces affirmations contradictoires, arrêtons-nous un instant sur cet aveu féminin qui appelle quelques réflexions.

Et tout d'abord une remarque métrique. Si l'on s'intéresse aux connexions métriques reliant les 13 sonnets de la tenson, on s'aperçoit qu'elles ne consistent en général qu'en une ou plusieurs assonances, sauf entre les sonnets 40-41 et 46-47 où la liaison est assurée dans les deux cas par deux rimes identiques ARE et ENTE qui sont parfois riches et qui peuvent reposer sur le même $\operatorname{mot}^{10}$. Or le sonnet 46 est le seul sonnet, avec le $n^{\circ} 40$ qu' on vient d'analyser, où la dame laisse entendre que son comportement $n^{\prime}$ est pas toujours conforme aux règles courtoises. En effet, après avoir évoqué la difficulté qu'aurait une dame "guère sage " à ne pas « commettre de folie » pour Guittone tant il « sait si subtilement persuader autrui » (v. 1-4), la dame affirme à ce dernier qu'en ce qui la concerne il ne pourra jamais la tromper, mais que cette impossibilité ne signifie pas qu'elle soit sage. Apporter une telle précision est une manière implicite de reconnaître qu'elle n'est pas sage:

Maintenant, je ne veux pas du tout dire que je sois sage ${ }^{11}$.

Ainsi, les sonnets de la dame les plus étroitement reliés à ceux de l'homme du point de vue métrique sont, semble$\mathrm{t}$-il, ceux dans lesquels elle montre ses défauts de courtoisie, c'est-à-dire ceux où elle se montre l'égale de l'amant déloyal et insincère qui est face à elle. Le fort accord métrique soulignerait ici la complicité morale entre les deux protagonistes. 
On voudrait faire une autre remarque sur les similitudes verbales existant entre des passages de la tenson et des passages de l'Art d'aimer de Guittone - connu depuis son édition par D'Arco Silvio Avalle sous le titre de Manuel du libertin ${ }^{12}$ - ainsi que sur les comparaisons que de telles similitudes permettent d'établir entre la (les) dame(s) du Manuel et la dame du dialogue 37-49. Certaines de ces similitudes verbales, précieusement relevées par L. Leonardi dans ses introductions et ses notes aux différents sonnets, laissent selon lui peu à peu entrevoir que la dame de la tenson participe au "gioco degli inganni " précisément conseillé dans le Manuel. Ce " jeu » consiste pour la dame courtisée à dire « non », alors qu'elle est d'accord et qu'elle fera le contraire de ce qu'elle dit. Pour l'homme il consiste à être conscient que ce " non " cache en réalité une acceptation, mais à faire semblant de ne pas en être conscient ${ }^{13}$. Selon L. Leonardi toujours, la duplicité de la dame, une sévérité de façade cachant sa complaisance, ne serait explicitement évoquée que dans la conclusion du sonnet 43 (v. 13-14). La lecture que nous venons de faire du sonnet 40 ainsi qu'une analyse des sonnets 14 et 15 du Manuel nous amènent d'une part à anticiper la révélation de la duplicité féminine, d'autre part à distinguer le comportement de la dame de la tenson de celui de la dame du Manuel. Il nous semble en effet que notre dame manifeste sa duplicité dès les tercets du sonnet 40 , là où elle dit qu'elle continuera à se montrer bienveillante envers son amant alors qu'elle sait qu'il n'est pas loyal. Elle va jusqu'à avouer elle-même cette duplicité, lui donnant de la sorte une publicité dont se garde au contraire la dame du Manuel, qui doit cacher sa complaisance par honte et peur du qu'en-dira-t-on. La dame de la tenson semble donc s'écarter plus du code de comportement courtois que celle de l'Art d'Aimer.
Revenons maintenant à la contradiction que nous avons mise en évidence entre les deux premiers sonnets dans lesquels la dame répond à Guittone. Nous avons vu que lorsqu'elle prend la parole pour la première fois elle accepte le dialogue et les services de son amant à condition qu'ils soient parfaitement courtois. Guittone cependant, dès le sonnet de réponse suivant (39), donne à sa dame un conseil qui contredit la prescription qu'il lui avait faite juste auparavant. Alors qu'il lui avait dit:

parce qu'on peut mentir et dire vrai,

n'ayez pas, sans preuve, foi en moi. (37, v. 3-4)

voici son conseil désormais:

Je vous conseille d'avoir vite, et sans crainte,

foi en mon amour et en ma fidélité. $(39, \mathrm{v}, 12-13)$

Cette contradiction masculine prouve d'emblée la mauvaise foi de Guittone et explique la diatribe contre les conseillers fallacieux. Elle devrait, selon toute logique, conduire la dame à abandonner toute bienveillance, une bienveillance qui se manifeste explicitement dans l'oreille attentive qu'elle prête à son amant et dans la réponse qu'elle accepte de lui donner. Le dialogue devrait donc s'interrompre à peine commencé, ce qui n'est pas le cas.

Comment expliquer cela? La duplicité de la dame ne nous paraît pas expliquer à elle seule la poursuite de ce dialogue hypocrite car, à l'inverse, dans la seconde moitié de la tenson, ce n'est pas sa droiture qu'elle allèguera pour justifier le congé qu'elle donne à Guittone (cf. 46, v. 5-8). En revanche on se demande s'il ne faut pas voir, dans la possibilité de la convaincre qu'elle laisse tout de même à Guittone, une occasion de faire fonctionner encore le discours courtois, de préserver son efficacité. Dans les 
exemples de dialogues entre une dame et son amant, fournis par André le Chapelain dans son De Amore cuvre que Guittone d'Arezzo connaissait bien, comme en témoigne le Manuel du libertin -, à plusieurs reprises les dames avaient poursuivi l'entretien alors qu'elles étaient conscientes du caractère insensé ou ambigu des propos de leur amant. Ainsi la dame du second dialogue répond à son interlocuteur, la première fois:

Mon âme supporte avec patience tes propos insensés et je te réponds avec aménité. (p. 62):

et la deuxième fois:

Tu essaies de masquer tes erreurs sous une telle faconde qu'il $\mathrm{m}^{\prime}$ est difficile de répondre à tes propos, pourtant parfaitement vains. Je tâcherai cependant de te réfuter en partie par le raisonnement. (p. 67)

De même la dame de la petite noblesse, si l'on en croit ce que lui dit le grand seigneur:

Bien que mes intentions transparaissent nettement au travers des termes voilés et des circonlocutions que j'ai employées, vous m'avez demandé de m'expliquer plus clairement sur mes desseins. (p. 104)

Tout le monde sait ce qui se cache sous le discours amoureux courtois. Ce qui importe dès lors est moins sa fonction stratégique que son agencement, " el modo de lo [...] parlare " dit la dame de la tenson $(40$, v. 13), la «copiosa sermonis facundia " dit André qui considère que $c^{\prime}$ est un des trois moyens qui permettent de gagner l'amour $^{15}$. Le noble qui dialogue avec une femme de la noblesse réussit à faire accepter ses services en temps et lieu, peut voir sa dame en chair et en os, parce qu'il connaît l'art du discours qui devient un mérite. Voici en effet comment sa dame justifie sa complaisance:

En vérité, tu as donné ses lettres de noblesse à l'art du discours, et c'est en toi que les mots charmeurs ont élu domicile, car tu as su présenter tes revendications avec infiniment de précautions et d'habileté. (p. 81)

L'amant Guittone n'est pas dépourvu de qualités oratoires: il sait, comme on l'a vu, " subtilement persuader autrui » $(46, v .4)$. Cependant, alors que dans le sonnet 42 la dame lui demande:

Démontre que tu as des raisons d'un autre genre iou : que tu as droit à un autre lieu] $(42, \text { v. } 7)^{10}$

dans le sonnet 43 , qui provoque le revirement cie la dame, il ne démontre pas ce qu'elle lui demande de démontrer. Il ne répond pas aux deux questions qu'elle lui pose (v. 14). Enfin, il détourne la conversation. Comme la dame il emploie le verbe " partire », seule connexion verbale avec le sonnet 42, mais il l'utilise différemment. La dame l'emploie comme un verbe transitif indirect et l'associe au discours - elle demande à Guittone, si jamais il a l'intention de demander à la voir dans un lieu secret, d'abandonner ce sujet - tandis que son interlocuteur lui donne son sens courant de verbe intransitif:

\section{Ora te parte ormai d'esta novella $(42$, v. 12)}

Bene vegg'io che di partir potenza/darmi potete (43, v. 9-10)

Guittone a ainsi complètement changé de sujet. Ce refus de répondre à sa dame est d'autant plus marquant que dans ses deux sonnets précédents (39 et 41) il s'appliquait au contraire à reprendre point par point ce qu'elle lui disait. 
Comparons d'abord les sonnets 38 (la dame) et 39 (Guittore). Dans les deux premiers vers la dame faisait allusion à son écoute et à sa réponse, une attitude qui selon elle prouvait qu'elle n'était pas «villana». Au même endroit et dans le même ordre, Guittone la remercie pour son " udienza " et son "responso " qu'il qualifie de "gente » (noble). Les vers 3 et 4 dans les deux sonnets se rapportent à la qualité de la réponse de la dame. Ce sont des commentaires qui amplifient le qualificatif " gente" ou " non... villana » au moyen d"une comparaison dont le comparant est identique (les autres dames). Le deuxième quatrain de Guittone dialogue avec le quatrain symétrique de son interlocutrice. Les deux ensembles se rapportent au discours de l'interlocuteur comme le montrent les verbes " dire " à la deuxième personne ${ }^{17}$ : " dici " $(38$, v. 5$)$ et « dite » $(39$, v. 5). La différence entre les deux passages est que Guittone explicite ce qui était implicite dans la mise en garde de la dame. Celle-ci en effet ne disait pas qu'elle allait agreer l'amour de son amant, mais qu'elle lui rendrait la monnaie de sa pièce: «... pour amour, amour je te rendrai, / mais, pour son contraire, ce qui est juste et équitable " (v. 7-8). Sa réponse en somme dépend de l'attitude de Guittone: c'est ni oui, ni non, d'où le " non... dite » (v. 5) et le " sì » (v. 7) employés par Guittone dans l'ordre inverse par rapport au contenu du discours de la dame. Le «per parola ornata " qualifie à juste titre la réponse de cette dernière qui a utilisé des hypothèses, c'est-à-dire des figures. Les vers 9-11 de Guittone contiennent une déclaration d'amour et de fidélité - au sens où il offre ses "bons et loyaux services" - qui reprend dans le même ordre les points du discours de son interlocutrice dans 38: "Amo » (39, v. 9) répond à " dici che m'ami " (38) et «fedel son d'ubidir" $(39$, v. 10$)$ à " fedel dare " et « fedel stare " (38). En outre, ce tercet insiste sur la fidélité ( ( fedel »v. 10 et « fede » v. 11) et sur l'amour qui grandit
(« avanza »), exactement comme le tercet correspondant dans la bouche de la dame (deux fois «fedel " aux vers 9 et 11 ; le verbe "cresce "). Enfin arrive le conseil de Guittone, dans des vers symétriques à ceux où la dame demandait conseil: en tête du vers 12 "consigliovo" répond à " consigliame » $(38$, v. 12$)$. Comparons maintenant les tercets du sonnet 40 (la dame) et les quatrains du sonnet 41 (Guittone) ${ }^{16}$. Les deux sentiments que l'amant dit éprouver (la douleur et la joie) naissent des deux affirmations de la dame: 41 v. 4 renvoie à 40 v. $7-10$, tandis que 41 v. $5-6$ « al modo de lo mio parlare » renvoie à 40 v. 13 "el modo de lo tuo parlare".

Tous ces rapprochements mettent en lumière le caractère particulier du sonnet 43 qu'on pourrait qualifier de " non réponse ". Ce refus implicite de répondre, de démontrer et d'argumenter contraste par ailleurs avec l'attitude des amants des dialogues du De amore: jamais ceux-ci ne refusent de répondre l'un à l'autre, et ils le font "systématiquement point par point sans fantaisie dans la démarche " et au point de donner " aux conversations l'allure d'argumentations d'école ${ }^{19}$ ". Par endroits ils se vantent même de leur argumentation. Voici ce que dit un grand seigneur:

J'ai donc détruit les deux arguments que vous avez présentés en leur opposant la meilleure des réponses et ils ne peuvent en aucune façon contrecarrer mes propos. ( $p$. 105)

Et une des réponses ultérieures de la dame montre combien la complaisance féminine dépend de l'argumentation masculine:

Vous n'avez, semble-t-il, exposé aucun argument qui puisse affaiblir mon opinion, ou $\mathrm{m}^{\prime}$ inciter à consentir à vos désirs. (p. 109) 
On peut dès lors émettre l'hypothèse que le revirement de la dame s'explique parce que Guittone a implicitement refusé de répondre à sa demande de démonstration. Elle lui avait offert (sonnet 40) la possibilité de la convaincre par le discours, mais dans le sonnet 43 il rompt ce pacte tacite.

Après cette forme d'interruption du dialogue que constitue la non-réponse de Guittone, on peut dire que s'ouvre une nouvelle tenson amorcée par la demande de conseil à la dame. Le dialogue reprend en effet, très serré, tant de la part de la dame qui va donner le conseil demandé, que de la part de Guittone qui va contester ce conseil point par point. Cependant la rupture est consommée et les arguments de Guittone, toujours contestés, ne parviennent à aucun moment à convaincre la dame.

Le conseil de cette dernière tombe comme un couperet au début du sonnet 44 ( "Je te conseille de partir " "). Il faut noter qu'elle a abandonné le tour qu'elle avait utilisé lors de sa précédente intervention (" te parte d'esta novella »), pour répondre strictement à Guittone en reprenant sa construction intransitive. Elle réutilisera dès lors systématiquement la forme «guittonienne », une nouvelle fois dans ce sonnet, puis dans chacune de ses allocutions suivantes: "parteti » $(44$, v. 9); « parteti ormai » $(46$, v. 10); "donque te parte $(48, v .9)$ dont le sens est confirmé par l'ultime injonction " or pensa di tener altro viaggio " (48, v. 12) ${ }^{21}$. Ce conseil s'oppose à celui que Guittone lui avait donné dans la première partie: à l'invitation à aimer, en laquelle se résume le tercet final du sonnet 39 , elle oppose une invitation à partir. Le mouvement de progression puis de régression du dialogue tout entier repose sur ces conseils opposés « aime/pars ».
Après le conseil initial, la dame reprend dans le même ordre que Guittone ce qu'il vient de lui dire: le « tu dis que » ouvrant les vers $2-4$ se réfère à ce que son amant disait dans les quatrains, tandis que le " et tu me dis que » ouvrant les vers $5-8$ se réfère au discours des tercets. Dans la première partie elle conteste d'abord son impuissance à partir en répondant par une figure dérivative, « podere » $(44, v .1)$, à ses « posso » et « potenza » (43, v.6 et 9), puis son attachement amoureux en variant " destretto " par " tenere »:

[...] tant elle m'a contraint à aimer (43, v. 4)

car je sais qu'amour ne doit pas avoir trop prise sur toi $(44, \text { v. } 3)^{22}$

Dans la seconde partie elle nie avoir le pouvoir que Guittone lui prête et dont il l'invite à user pour faciliter son départ: le pouvoir de s'enlaidir. Le dialogue se noue, ici encore, grâce à des figures dérivatives. Guittone prétend qu'elle peut l'aider à partir ainsi :

Rien qu'en rendant moins belle votre beauté

et en disant et faisant ce qui convient pour déplaire. (43, v. 11-12).

\section{Elle lui répond :}

Tu me dis de m'efforcer de déplaire

rien que pour paraître avoir le cœur épris :

mais cela ne t'est pas nécessaire: je ne suis pas belle

au point de pouvoir rendre quelqu'un amoureux de moi.

$(44, \text { v. } 5-8)^{23}$.

Dans le sonnet 45 Guittone va contester à son tour ce qu'elle a dit sur son attachement amoureux à lui et sur sa beauté à elle. Pour revenir sur l'amour qu'il lui porte, il 
allie deux techniques: il développe le champ lexical du lien $[$ destretto $(43)>$ tenere $(44)>$ stringe $(45, v .11)]$ et il crée un nouveau lien au moyen de l'antithèse « falso amante/fino amante ». Sa dame lui avait adressé l'accusation suivante:

Tu es un faux amant et un simulateur. (44, v. 4).

Il prétend au contraire:

\section{(...) Je suis un parfait amant. $(45, \text { v. } 6)^{24}$}

En ce qui concerne la beauté de la dame il joue sur la synonymie " piacere = bellezza ", comme elle l'avait déjà fait, et crée un lien au moyen de l'opposition "laida/ bella ». La dame avait dit d'elle-même « je suis laide » (44, v. 11), il rétorque:

Et si vous n'êtes pas belle, moi, pourtant, je vous tiens pour beaucoup plus belle que toute autre $[\ldots]^{25}$.

Enfin il reprend son discours sur la parité sociale: elle l'avait invité à aimer une dame qui soit son égale $(44$, v. 9 $10)$, il ne prétend pas à être aimé en égal (45, v. 12)

Les arguments que la dame oppose ici à Guittone, tout comme ceux de Guittone, ne sont pas originaux. On les trouve notamment dans les dialogues du De amore dont notre tenson reflète aussi la rigueur de l'argumentation.

C'est le cas du discours sur la beauté. La dame de la tenson (44, v. 11) et la femme du premier dialogue (p. 55) où s'entretiennent un roturier et une roturière, disent qu'elles ne sont pas belles. Chaque amant rétorque que si son aimée n'est pas belle, le fait même qu'il la tienne pour plus belle que toute autre femme prouve la réalité de son amour. Chez Guittone d'Arezzo les derniers vers que l'on a cités se poursuivent de la manière suivante:

[...] cela vous prouve

qu'amour m'étreint plus que je ne vous le montre. $(45, \mathrm{v}$. $10-11)^{20}$.

Tandis qu'André fait dire à l'homme:

Et même si tu crois que tu n'es pas belle, sois persuadée de la vérité de mon amour puisque, pour moi, ta beaute l'emporte sur celle de toutes les autres femmes. (p. 55)

L'hypothèse qui clôt le sonnet 44 :

Et sache que, si je devais aimer

ce n'est pas toi que j'aimerais (ne le prends pas mal) (v 12-13) ${ }^{2}$

fait écho à une hypothèse similaire émise par la dame de haute noblesse dans le dernier dialogue proposé par André (p. 118)

Cette hypothèse paraît rejetée dans le sonnet 46 :

bien que tu sois, des trois, celui que

j'aime plus que tout, foi que je dois à Dieu!:

mais non pas de l'amour auquel tu penses. (v. 12-14) ${ }^{28}$

mais on sait depuis le sonnet 40 que personne n'est de bonne foi. Quoi qu'il en soit l'aveu d'un sentinent distinct de ce que l'homme a en tête figure aussi dans le De amore. La femme de la noblesse qui s'entretient avec un noble lui avoue toute sa reconnaissance, lui promet ses pensées et lui permet de la voir en chair et en os, mais l'avertit qu'il ne parviendra pas à obtenir ce qu'il demande (p. 81-82). 
L'argument qui va mettre un terme définitif à la tenson - le libre arbitre féminin - recueille aussi l'approbation commune des deux interlocuteurs du second dialogue d'André. Guittone ne parviendra pas à séduire sa dame:

car une telle chose ne fut ni ne sera

jamais à (son) gré, en aucune manière. (v. 7-8)

Telle est sa volonté ( il voler (suo)», v. 9) et Guittone ne doit pas espérer lui faire changer d'avis (48, v. 9-11). La femme, puis l'homme du second dialogue partagent ce point de vue. Elle dit:

A mour a laissé à toute femme la liberté d'aimer le prétendant qui lui plaît et de repousser celui qui ne lui plaît pas. (p.63)

Il confirme:

Amour a laissé à la femme la liberté de choix: quand on l'aime, elle peut aimer en retour, si elle veut, si elle ne le veut pas, rien ne l'y force. (p. 66)

La tenson s'achève sur le refus implicite de la dame de répondre aux trois questions que lui pose Guittone:

Comment puis-je abandonner mon amour pour vous, objet unique capable de me donner la joie?

Comment puis-je persévérer, puisque cela ne peut vous plaire,

mais vous paraît, comme vous me dites, pénible au contraire?

Mais si je vous déplais, hélas, en vous servant,

vous plairai-je, par hasard, en ne vous servant pas ${ }^{20}$ ?
Ces questions topiques sont à la fois rhétoriques et réelles. Elles constituent une sorte de demande de conseil à laquelle la dame répond par une autre question, autrement dit à laquelle elle ne répond pas. Dans le sonnet 43 Guittone avait adopté la même attitude à l'égard des deux questions de la dame: il n'y avait pas répondu et avait posé une autre question. Mais à la différence de ce qui se produit maintenant, cette question avait suscité la demande de conseil qui avait ouvert la "deuxième " tenson. Ici, singulièrement, la dame souhaite désormais le conseil de Dieu: autre manière de mettre un terme au dialogue, qui s'ajoute au refus littéral de poursuivre le débat, et à la reprise de la rime en AGGIO par laquelle la dame avait commencé à répondre à Guittone ${ }^{3 i}$.

Et que Dieu me conseille en tout

comme je l'ai fait avec toi en cette occasion. $(48,7-8)^{31}$

$\star \star \star *$

Loin d'éclaircir toutes les obscurités de cette tenson et de mettre à jour toute la logique d'un dialogue sans cesse miné par la mauvaise foi des interlocuteurs, cette étude permet du moins de comprendre pourquoi la dame prolonge l'entretien alors que la condition de franchise qu'elle a posée au départ n'est pas respectée, et pourquoi le sonnet 43 provoque sa volte-face.

La duplicité du langage courtois étant vite établie, ce n'est pas la vérité qui sert à convaincre la dame d'être bienveillante avec son amant, mais l'aptitude de ce dernier à argumenter. Ce n'est pas nouveau : quel que soit son sujet une tenson repose toujours sur cet art du discours. Mais dans le cas précis d'une tenson où un amant vise à se faire aimer d'une dame, cet art est encore plus indispensable: c'est ce qu'enseigne André le Chape- 
lain pour qui "l'extrême facilité d'élocution" (copiosa sermonis facundia) constitue un des trois moyens de gagner l'amour. Les dialogues du De amore illustrant cet art sont d'ailleurs, comme on l'a vu, une source non négligeable pour la tenson de Guittone, à cette différence près que cette dernière exclut nécessairement tout examen de cas de figure sociaux. A la différence, aussi, qu'elle coule le débat dans le moule du sonnet qui, grâce à sa métrique, renforce le jeu de l'argumentation. A l'énorme différence encore que les interlocuteurs de la tenson sont totalement dépourvus de « l'excellente moralité » chère au Chapelain. Ce défaut les assimile aux amants d'une autre "série " de Guittone: la série qu'Avalle a appelée Manuel du libertin dont les vers ont plus $d^{\prime} u n$ point commun avec ceux de la tenson mais dans laquelle on retrouve la « hiérarchie » des interlocuteurs du De amore absente de cette dernière. Ce qui distingue également les deux groupes de sonnets, $c^{\prime}$ est le personnage de la dame. Prenant directement la parole dans la tenson, tandis qu'elle n'est que l'objet du discours masculin dans le "Manuel ", elle manifeste une duplicité sans état d'âme, insouciante d'un qu'en-dira-t-on que la quasi inexistence de la société rend improbable.

Anne ROBIN

\section{NOTES}

1. GUITTONE D'AREZZO, Canzoniere I sonetti d'amore del codice laurenziano, a c. di Lino Leonardi, Turin, Finaudi, 1994. Je me référerai à cette édition, avant laquelle on considérait déjà que la tenson faisait partie d'un cycle comprenant les sonnets 31 à 80 . Cf. Claude MARGUERON, Recherches sur Guittone d'Arezzo, Paris, PUF, 1966, p. 403.

2. Cf. les caractéristiques du genre tenson définies par Michelangelo PICONE, " La tenzone De Amore fra Iacopo Mostacci, Pier della Vigna e il notaio », in Il genere « tenzone " nelle letterature romanze della Origini, Ravenne, Longo, 1999, p. 17-18.

3. La tenson entre Guittone et Mastro Bandino, qui occupe les sonnets 28-30 de l'édition Leonardi, constitue un autre exemple de ces demandes de conseil topiques concernant un comportement amoureux. Cf. en outre l'introduction au sonnet 38. De même lorsque dans la célèbre tenson « De amore " lacopo Mostacci veut entendre l'avis de ses interlocuteurs à propos de la nature d'amour, et lorsque Dante sollicite l'avis de " toutes les âmes éprises et tous les nobles cœurs », on est toujours en présence de demande de conseil.

4. Dici che m'anni forte a bon coraggio: or mira bene se la parola è satun.

ché per amor, amor ti renderaggio,

e, del contraro, ciò ch ê ragion certana.

5. Consigliame, com'on leale e saggio,

ch 'eo deggia ver'del tuo dimando fare, ché de leal consiglio non partraggio.

6. L'insistance avec laquelle il est rappelé ici s'explique peutêtre aussi par un épisode de l'histoire que L. Leonardi met en évidence dans le canzoniere: après avoir initialement eu un comportement que le personnage Guittone a proposé luimême comme un exemple de courtoisie, ce dernier a avoué que ce comportement n'était pas loyal ni honnête (sonnet 19). 
Mais notre objet d'étude étant la seule tenson, nous nous abstiendrons désormais d'établir des liens avec ce qui précède ou ce qui suit.

7. Avec un vocabulaire portant beaucoup plus l'empreinte de la topique courtoise, cette diatribe fait écho à un passage du dialogue $\mathrm{H}$ du De Amore " entre un grand et une dame de la haute noblesse »: « La femme: Tout homme essaie d'amener une personne réticente à consentir à ses désirs les plus profonds en utilisant tous les arguments possibles. Mais on ne peut tenir pour ami quelqu'un qui, en nous donnant des conseils, ne cherche que son propre intérêt et ne se préoccupe point de nous venir en aide, et l'on ne doit point suivre les désirs d'un ami qui n'a en vue que son profit ". ANDRE LE CHAPELAIN, Truité de l'amour courtois, traduction Claude Buridant, Paris, Klincksieck, 1974, p. 124.

8. “Colore" à la rime v. 8 et « colorare " à la rime dans le vers suivant.

9. Ma non te pòi ver'me si colorare ch'e'ben non te conosca apertamente, avegua ch'eo però non voi'lassare ched eo non te recera a benrogliente secondo el modo de lo tuo parlare, ad intenderlo pur sinplicintente.

10. colorARE: lassARE: parlARE $(40$, v. $9,11,13)$; contARE: penSARE: parlARE $(41$, v. $2: 4: 6: 8)$; apertamENTE: benvogliENTE: sinplicimENTE $(40$, v.10: 12:14) et pienamENTE, pienamENTE, gENTE $(41$, v.10:12:14) et folleggiARE: sermonARE: 'ngegnARE: affARE (46, v:2: 4:6 8); stARE: donARE: amARE: pare $(47$, v. $2: 4: 6: 8$ ); gravemENTE: saccENTE: $n E N T E$ : convenENTE $(46$, v. $1: 3$ 5:7); noiosamENTE: spiacENTE: piacENTE (47, v. 9: $11: 13$ )

11. Or non voi'dire ch'eo sia saggia nente (v. 5).

12. Ai luoghi di delizin pieni. Saggio sulla lirica italinna del XIII secolo, Milan-Naples, Ricciardi, 1977, p. 163-187.
13. Cf. sonnets $n^{\circ} 100$ et 101 in Le rime di Guittone d'Arezzo, a cura di Francesco Egidi, Bari, Laterza, 1940, correspondant aux $\mathrm{n}^{\circ} 14$ et 15 de Avalle qui détaille ce jeu, in op. cit., p. 76-78.

14. 37: Però c'omo mentir e dir ver osa, for prova, non abbiate in me credenza.

39: Consigliovo che tosto, e non dottando, de mi'amar e de mia fé fidanza prendiate $[\ldots]$.

15. Op. cit., p. 52.

16. Dimostra se ragion ài d'altro lato. «Altro lato", syntagme également présent dans un sonnet du Manuel, est ambigu. Dans le Martuel (15, v. 6) Avalle propose de garder l'ambiguïté, d'où mes deux traductions.

17. La dame tutoie son amant vassal qui vouvoie sa dame suzeraine. Cet usage est traditionnel.

18. La première partie du sonnet de la dame est une diatribe impersonnelle qui relève du monologue et non du dialogue. La deuxième partie du sonnet de Guittone est une requête qui introduit nécessairement du nouveau.

19. Cl. BURIDANT, op. cit., Introduction, p. 16.

20. Consiglioti che parti. (44, v. 1).

21. Pense désormais à suivre une autre voie.

22. I...I sì $m \hat{a}$ destretto amando. (43) ch'eo so ch'amor non tá troppo a tenere. (44)

23. Guittone: sol con disabellir vostra piagenza e dir e far ciò c'a spiacer pertene.

La dame: e dicimi ch'io peni a dispiacere sol per parer d'innamorato core; e opo non $t \hat{e}$ : non son de tal piacere ch'e'far potesse de me amadore.

24. La dame: anzi se'falso amante e 'nfingitore. Guittone: $[$... ben fino amante eo sono $[. . . l$.

25. La dame: laida son.

Guittone: E, se bella non sete, ed eo vi tegno più bella c'altra assai $[$...]. 
26. [...] per ciò provate c'amor mi stringe più ch'eo non v'asegno.

27. E sappeti che, s'eo dovesse amare, eo non ameria te (non l'abbi a male).

28. tutto sie tu, dei tre l'un ch'amo assai più che cos'altra mai, fé che deo a Deo! ma non de quello amor che penser ài.

29. Partire con'poss'eo d'amar voi, cosa sola sete ' $n$ potermi gioi donare? Istar con'posso, poi voi piacer no osa, ma sì noioso, me dite, vo pare? Ma se vi spiaccio, lasso, per servire, serò per deservir, forse, piacente?

30. Indiqué par L. Leonardi: Eo t'aggio inteso, e te responderaggio $(38, \mathrm{v} .1)$ Certo sii ch'esta volta è la poi

ch'e'd'esto fatto ormai te parleraggio. (48, v. 13-14)

31. e si consigli me Deo in ogne lato, com'eo fatt'aggio te ' $n$ esto misteri. $(48, v .7-8)$ 